\title{
Routine Inferior Vena Cava Filter Retrieval: A Cost Minimization Analysis of Loop Snare Versus Rigid Endobronchial Forceps Assisted Retrieval Techniques
}

John Swietlik ( $\sim$ jswietlik@uwhealth.org)

University of Wisconsin https://orcid.org/0000-0002-7180-794X

Quinn Steiner

University of Wisconsin-Madison School of Medicine and Public Health

Paul F Laeseke

University of Wisconsin Hospital and Clinics

Aaron Eifler

University of Wisconsin Hospital and Clinics: University of Wisconsin Hospitals and Clinics

\section{Short Report}

Keywords: Venous thromboembolism, IVC filter retrieval, Loop-snare, Endobronchial forceps, Cost minimization analysis

Posted Date: January 20th, 2022

DOI: https://doi.org/10.21203/rs.3.rs-1233158/v1

License: (c) (1) This work is licensed under a Creative Commons Attribution 4.0 International License.

Read Full License 


\section{Abstract}

Routine inferior vena cava filter (IVCF) retrieval is a common procedure that can be accomplished with different techniques. Simple loop snare retrieval is common; however, snares are single use and many seemingly routine retrievals require more advanced techniques. Rigid endobronchial forceps (EF) retrieval is an effective advanced approach with the ability for the forceps to be sterilized and re-used for multiple procedures. The purpose of this study was to compare the cost effectiveness of snare versus forceps IVCF retrieval techniques at a single institution.

This study is a cost minimization analysis including an IRB approved, single-center, retrospective review of all IVCF retrievals from January 2019 to April 2021. Routine IVCF retrieval techniques were categorized as loop snare-only retrieval (SR), forceps-assisted after unsuccessful snare retrieval (S+F), and forcepsonly retrieval (FR). Total equipment cost for each technique was determined.

Seventy-seven successful routine IVC filter retrieval procedures were performed at our institution during the study period ( $63 \mathrm{SR}, 8 \mathrm{~S}+\mathrm{F}, 6 \mathrm{FR}$ ). There were no technical failures. FR was the most cost-effective technique with per-procedure equipment cost of SR, S+F, and FR techniques of $\$ 434.30, \$ 556.55, \$ 221.98$ respectively. Average equipment cost for the 77 retrievals over the study was $\$ 430.46$. Had all retrievals been performed using the FR technique, average equipment cost per retrieval would have decreased by $\$ 208.48$, for a total cost savings of $\$ 16,052.72$.

Utilization of FR technique for all routine IVCF retrievals would result in a meaningful cost savings compared to standard SR and S+F techniques.

\section{Introduction}

Venous thromboembolism (VTE) is a significant cause of morbidity and mortality. Although anticoagulation is first line therapy, IVC filters (IVCF) remain a mainstay in therapy for patients with VTE who have contraindications to or have failed anticoagulation [1-3]. IVCFs were originally placed with the intention of long-term use, however in 2010 the FDA released a recommendation that IVCFs should be removed as soon as medically appropriate. This recommendation was reiterated by the FDA in 2014 and more recently has been reflected in current practice guidelines made in collaboration with multiple medical societies $[4,5]$. These recommendations have been the major driver of increasing IVCF retrievals.

The use of a simple loop-snare is among the most common IVCF retrieval techniques. Due to the number of patients with chronically indwelling IVCF now undergoing filter retrieval there have been significant advances in retrieval techniques. Although effective in many routine IVCF retrieval procedures, the use of a loop-snare has the drawback of being single use [6, 7]. Additionally, advanced techniques are often required following loop-snare technique failures [6-8]. Rigid endobronchial forceps (EF) retrieval has been developed for use in chronically embedded IVCFs. EF have also been used during routine IVCF retrieval cases after standard techniques have failed [6-8]. EF can be sterilized and re-used for multiple procedures. Given the increase in retrieval rates, it has become increasingly relevant to consider the 
financial implications of the varying retrieval techniques, but data evaluating the cost of IVCF retrieval techniques is currently lacking. The purpose of this study was to compare the cost effectiveness of EF forceps-only versus simple loop snare techniques for simple IVCF retrievals at a single institution.

\section{Methods}

Institutional review board approval was obtained to perform a retrospective review of all routine IVCF retrievals performed during a 27-month period (1/1/2019 to 4/30/2021) at a single institution. Epic HealthLink (Epic Systems Corporation, Verona WI) was queried using procedure codes for all IVCF retrievals. A total of 94 procedures were identified. Chronic (>12-month dwell time) and complex filter retrievals (defined by pre-procedural imaging findings) were excluded. A total of 77 routine IVCF retrieval procedures were included for review. The procedures were subcategorized based on retrieval technique. Routine IVCF retrieval techniques were categorized as loop snare-only retrieval (SR), forceps-assisted after unsuccessful snare retrieval ( $\mathrm{S}+\mathrm{F})$, and forceps-only retrieval (FR). An institutional procedural database was reviewed for all 77 cases. Procedural equipment for all cases were recorded, and a standard procedural equipment setup was created from the most used equipment for each technique. Total equipment cost for the standard procedural equipment of each technique was calculated based on product specific company prices. The EF used for filter retrieval can be autoclaved between cases for repeat use in multiple procedures. Therefore, an estimated per procedure cost was calculated for inclusion in the study. For the purpose of this study the EF was given a conservative shelf life of 2 years based on potential wear and tear, as well as the theoretical risk for the EF to be misplaced or lost. In review of the current study period, 75 IVCF retrievals were completed over a 2-year period. Hence, a per procedure cost of $\$ 14$ was calculated based on the total cost $(\$ 1050)$ divided by the procedures completed within a 2-year timeframe. All other equipment used was single use and the included cost was based off company prices. Cost analysis was then performed comparing the varying retrieval techniques. Chart review was completed for each case to determine procedural technical success and procedural complications. Technical success was defined as complete removal of the IVC filter. Procedural complications were based on the Society of Interventional Radiology (SIR) adverse event classification [9].

\section{Results}

Seventy-seven successful routine IVCF retrieval procedures were performed at our institution during our period of study ( 63 SR, 8 S+F, 6 FR). Technical success was $100 \%$ for all procedures. Table 1 summarizes the cost of the procedural equipment for each technique. FR was the most cost-effective technique with a per procedure equipment cost of $\$ 221.98$, compared to $\$ 434.30$ for SR, and $\$ 556.55$ for $\mathrm{S}+\mathrm{F}$. Average equipment cost for the 77 retrievals over the study period was $\$ 430.46$, for a total cost of $\$ 33,145.42$ for the 27-month period. Had all retrievals been performed using the FR technique, average equipment cost per retrieval would have been decreased by $\$ 208.48$ for a total cost savings of $\$ 16,052.72$ over the 27 - 
month period (see Table 2 for summary of the cost comparison of retrieval techniques). This potential cost savings represents $48 \%$ of the total procedural equipment cost.

Table 1

Summary of Equipment Cost

\begin{tabular}{|c|c|c|}
\hline Snare only technique & $\begin{array}{l}\text { Snare }+ \text { forceps assisted } \\
\text { technique }\end{array}$ & Forceps only technique \\
\hline Micropuncture set $(\$ 27.00)$ & Micropuncture set $(\$ 27.00)$ & Micropuncture set $(\$ 27.00)$ \\
\hline Amplatz wire $(\$ 45.20)$ & Amplatz wire (\$45.20) & Amplatz wire (\$45.20) \\
\hline Pigtail flush catheter $(\$ 22.28)$ & Pigtail flush catheter $(\$ 22.28)$ & Pigtail flush catheter $(\$ 22.28)$ \\
\hline $\begin{array}{l}10 \text { Fr Check-Flo catheter } \\
(\$ 68.00)\end{array}$ & $\begin{array}{l}10 \mathrm{Fr} \text { Check-Flo catheter } \\
(\$ 68.00)\end{array}$ & $12 \& 14 \mathrm{Fr}$ dilators $(\$ 10.50)$ \\
\hline \multirow[t]{6}{*}{ EnSnare (\$271.82) } & EnSnare $(\$ 271.82)$ & $\begin{array}{l}16 \text { Fr Check-Flow catheter } \\
(\$ 103.00)\end{array}$ \\
\hline & 14 Fr dilator $(\$ 5.25)$ & Endobronchial Forceps \\
\hline & & (\$14 per use) \\
\hline & $\begin{array}{l}16 \text { Fr Check-Flow catheter } \\
(\$ 103.00)\end{array}$ & \\
\hline & Endobronchial Forceps & \\
\hline & (\$1050->\$14 per use) & \\
\hline Total Cost: $\$ 434.40$ & Total Cost: $\$ 556.55$ & Total Cost: \$221.98 \\
\hline
\end{tabular}

Table 2

Cost comparison of Retrieval Techniques

\begin{tabular}{|lllll|}
\hline $\begin{array}{l}\text { Retrieval } \\
\text { technique }\end{array}$ & $\begin{array}{l}\text { Avg } \\
\text { total } \\
\text { cost }\end{array}$ & $\begin{array}{l}\text { Cost comparison to } \\
\text { primary forceps } \\
\text { retrieval }\end{array}$ & $\begin{array}{l}\text { No. of IVC filter removal } \\
\text { procedures completed }\end{array}$ & $\begin{array}{l}\text { Potential cost } \\
\text { savings of forceps } \\
\text { retrieval }\end{array}$ \\
\hline FR & $\$ 221.98$ & - & 6 & - \\
\hline$S R$ & $\$ 434.30$ & $+\$ 212.32$ & 63 & $\$ 13,376.16$ \\
\hline$S+F$ & $\$ 556.55$ & $+\$ 334.57$ & 8 & $\$ 2,676.56$ \\
\hline Total & & & 77 & $\$ 16,052.72$ \\
\hline
\end{tabular}

\section{Discussion}

This study demonstrated that the primary use of EF for routine IVCF retrieval procedures allows for a procedural cost reduction compared to a snare-loop retrieval technique. Additionally, given that in IVCF 
retrieval cases where standard snare-loop retrieval fails, EF may be needed to achieve technical success, primary use of EF would eliminate cost of using both techniques. The equipment cost reduction per retrieval is substantial and can be impactful when extrapolated over time. This cost savings becomes increasingly meaningful with the increasing number of IVCF retrievals that are being performed annually throughout the US.

Chronic indwelling IVCFs have potential complications including filter fracture, device migration, organ penetration, IVC thrombosis, and increased DVT risk [7, 10, 11]. In 2010 the FDA issued a recommendation that all IVCFs be removed as soon as medically appropriate and that physicians who place IVCF ensure appropriate management of retrieval [5]. This recommendation has led to an increase in IVCF retrievals, including complex retrievals due to chronically indwelling and embedded IVCF. Increased IVCF dwell times have been demonstrated to increase IVCF retrieval failure [8, 12]. The large patient population with chronic indwelling IVCF requiring complex retrieval led to a need for development of new retrieval techniques, including the EF [7-9]. The EF can be advanced through a large caliber venous access sheath (figure 1: add in picture of forceps). The EF is used to grasp the filter hook, then the sheath is advanced over the EF while appropriate tension is kept on the EF which forces the IVCF to collapse into the sheath. The IVCF can then be removed through the sheath. The EF can be used for blunt dissection of embedded filter hook if needed, and the curvature of the EF can be manipulated to reach a tilted and embedded filter when necessary [6, 7]. As proceduralist have become more experienced and skilled with EF their use has expanded beyond complex IVCF retrievals. EF have potential benefits when used in routine IVCF retrieval cases. The current study demonstrated a meaningful potential equipment cost savings when primarily using EF retrievals for all routine IVCF retrievals. Although the current study demonstrated $100 \%$ technical success and no complications with the use of FR, future studies over a larger cohort are required to evaluate technical success and procedural complications rates.

The current study has multiple limitations. The single institution retrospective design of this study may limit its application. Additionally, the EF retrieval technique is user dependent. Proceduralists who perform IVCF retrievals may have less familiarity with the EF and be less proficient than those in the current study which might impact the widespread application of EF. However, as proceduralists complete greater numbers of complex IVCF retrievals the use of EF will become more ubiquitous. At the study institution the EF can be autoclaved at no direct cost to the department, however this may not be the case at other institutions which may decrease the potential cost savings. Although this study demonstrates a potential equipment cost savings from the use of a primary EF technique for all routine IVCF retrievals, further studies are needed to determine any differences in fluoroscopy time and radiation exposure (both to patient and physician) compared to standard snare-loop techniques.

\section{Conclusion}

Primary utilization of FR for all routine IVCF retrievals could result in a meaningful cost savings compared to current standard techniques. 


\section{List Of Abbreviations}

IVCF

Inferior vena cava filter

$\mathrm{EF}$

Endobronchial forceps

SR

Snare-only retrieval

$\mathrm{S}+\mathrm{F}$

Snare and forceps retrieval

FR

Forceps-only retrieval

\section{Declarations}

Ethics approval and consent to participate: Institutional review board approval was granted for this retrospective review.

Consent for publication: Given the retrospective nature of the study and that no specific patient data was included no consent was required by the institution.

Availability of data and material: The datasets used and/or analyzed during the current study are available from the corresponding author on reasonable request.

Competing interests: The authors JFS, QS, and ACE declare that they have no conflict of interest. PFL has research grants from Siemens Medical and HistoSonics; is a paid consultant for Ethicon, HistoSonics, and Elucent Medical; and is a stockholder in Elucent Medical, McGinley Orthopedic Innovations, and Histosonics, Inc.

Funding: This study was not supported by any funding.

Authors' contributions: JFS and ACE completed all data retrieval and interpretation. JFS and QS were major contributors in writing the manuscript. All authors read and approved the final manuscript.

Acknowledgements: Not applicable.

\section{References}

1. Angel LF, Tapson V, Galgon RE, Restrepo MI, Kaufman J (2011) Systematic review of the use of retrievable inferior vena cava filters. Journal of Vascular and Interventional Radiology: JVIR 22(11):1522-1530e3. https://doi.org/10.1016/j.jvir.2011.08.024

2. Kearon C, Akl EA, Ornelas J, Blaivas A, Jimenez D, Bounameaux H, Huisman M, King CS, Morris TA, Sood N, Stevens SM, Vintch JRE, Wells P, Woller SC, Moores L (2016) Antithrombotic Therapy for VTE 
Disease: CHEST Guideline and Expert Panel Report. Chest 149(2):315-352.

https://doi.org/10.1016/j.chest.2015.11.026

3. Sarosiek S, Crowther M, Sloan JM (2013) Indications, complications, and management of inferior vena cava filters: The experience in 952 patients at an academic hospital with a level I trauma center. JAMA Internal Medicine 173(7):513-517. https://doi.org/10.1001/jamainternmed.2013.343

4. Kaufman JA, Barnes GD, Chaer RA, Cuschieri J, Eberhardt RT, Johnson MS, Kuo WT, Murin S, Patel S, Rajasekhar A, Weinberg I, Gillespie DL, American College of Surgeons Committee on Trauma, American Heart Association (2020) Society of Interventional Radiology Clinical Practice Guideline for Inferior Vena Cava Filters in the Treatment of Patients with Venous Thromboembolic Disease: Developed in collaboration with the American College of Cardiology, American College of Chest Physicians, Society for Vascular Surgery, and Society for Vascular Medicine. Journal of Vascular and Interventional Radiology: JVIR, 31(10), 1529-1544. https://doi.org/10.1016/j.jvir.2020.06.014

5. United States Food and Drug Administration. Alerts and notices (medical devices) removing retrievable inferior vena cava filters: initial communication

6. Daye D, Walker TG (2017) Novel and Advanced Techniques for Complex IVC Filter Retrieval. Curr Treat Options Cardiovasc Med 19(4):28. https://doi.org/10.1007/s11936-017-0529-3

7. Desai KR, Pandhi MB, Seedial SM, Errea MF, Salem R, Ryu RK, Lewandowski RJ (2017) Retrievable IVC Filters: Comprehensive Review of Device-related Complications and Advanced Retrieval Techniques, vol 37. A Review Publication of the Radiological Society of North America, Inc, Radiographics, pp 1236-1245. 4https://doi.org/10.1148/rg.2017160167

8. Defining Prolonged Dwell Time: When Are Advanced Inferior Vena Cava Filter Retrieval Techniques Necessary? (n.d.). https://doi.org/10.1161/CIRCINTERVENTIONS.116.003957

9. Khalilzadeh O, Baerlocher MO, Shyn PB, Connolly BL, Devane AM, Morris CS, Cohen AM, Midia M, Thornton RH, Gross K, Caplin DM, Aeron G, Misra S, Patel NH, Walker TG, Martinez-Salazar G, Silberzweig JE, Nikolic B (2017 Oct;28(10):1432-1437.e3) Proposal of a New Adverse Event Classification by the Society of Interventional Radiology Standards of Practice Committee. J Vasc Interv Radiol. doi: 10.1016/j.jvir.2017.06.019. Epub 2017 Jul 27. Erratum in: J Vasc Interv Radiol. 2018 Jan;29(1):146. PMID: 28757285

10. Al-Hakim R, Kee ST, Olinger K, Lee EW, Moriarty JM, McWilliams JP (2014) Inferior vena cava filter retrieval: Effectiveness and complications of routine and advanced techniques. Journal of Vascular and Interventional Radiology: JVIR, 25(6), 933-939; quiz 940.

https://doi.org/10.1016/j.jvir.2014.01.019

11. Inferior Vena Cava (IVC) Filters-Risk of Serious Complications. (n.d.). Retrieved January 26 (2021) from https://healthycanadians.gc.ca/recall-alert-rappel-avis/hc-sc/2016/59518a-eng.php

12. Jaberi A, Tao MJ, Eisenberg N, Tan K, Roche-Nagle G (2020) IVC filter removal after extended implantation periods. The Surgeon: Journal of the Royal Colleges of Surgeons of Edinburgh and Ireland 18(5):265-268. https://doi.org/10.1016/j.surge.2019.10.003 
Figures

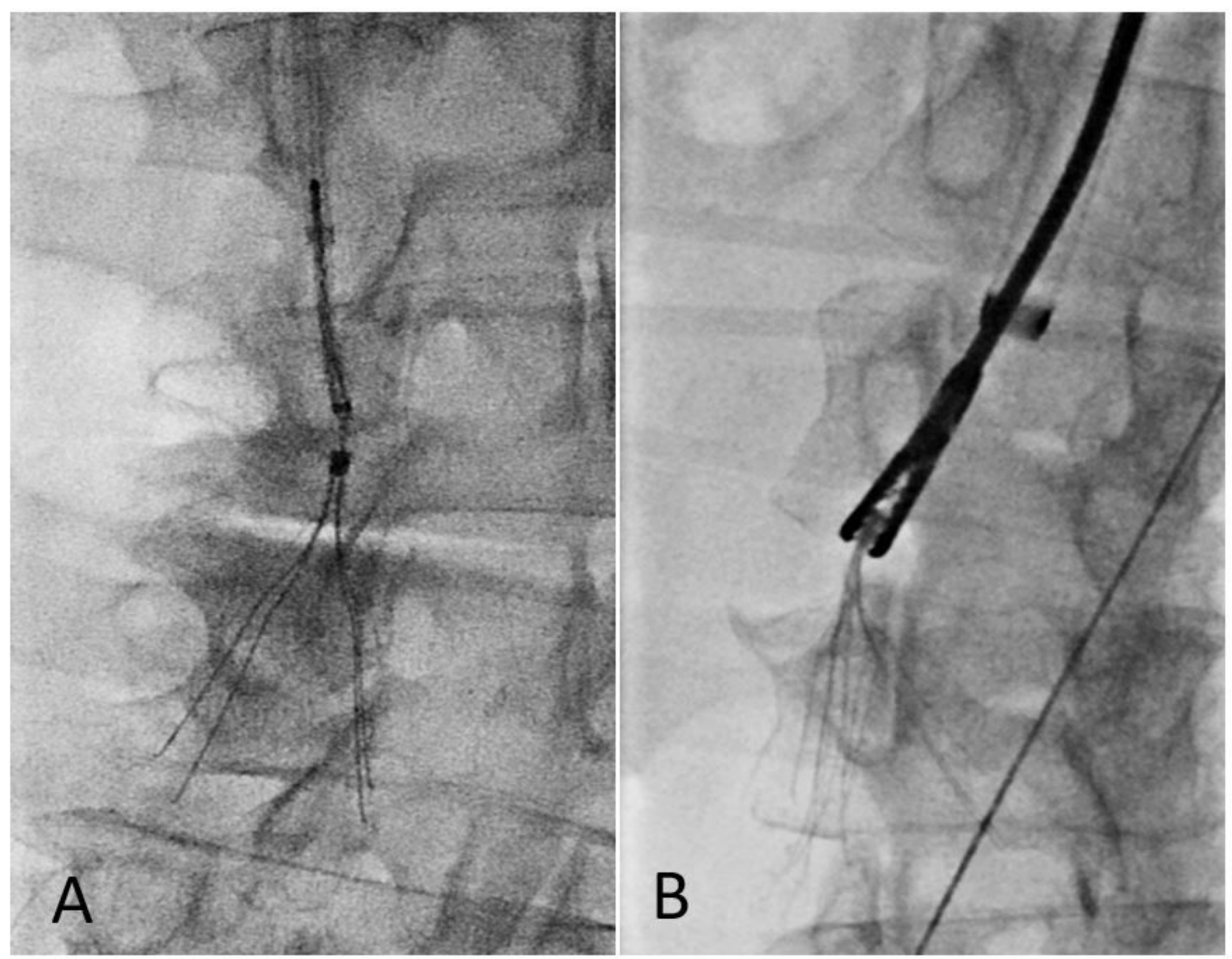

\section{Figure 1}

Routine IVCF retrieval can be accomplished with different techniques. Simple loop snare retrieval (A) is a common approach. However, REF retrieval (B) is a new technique with increased application for IVCF retrieval with the added advantage that they can be sterilized and re-used for multiple procedures. 


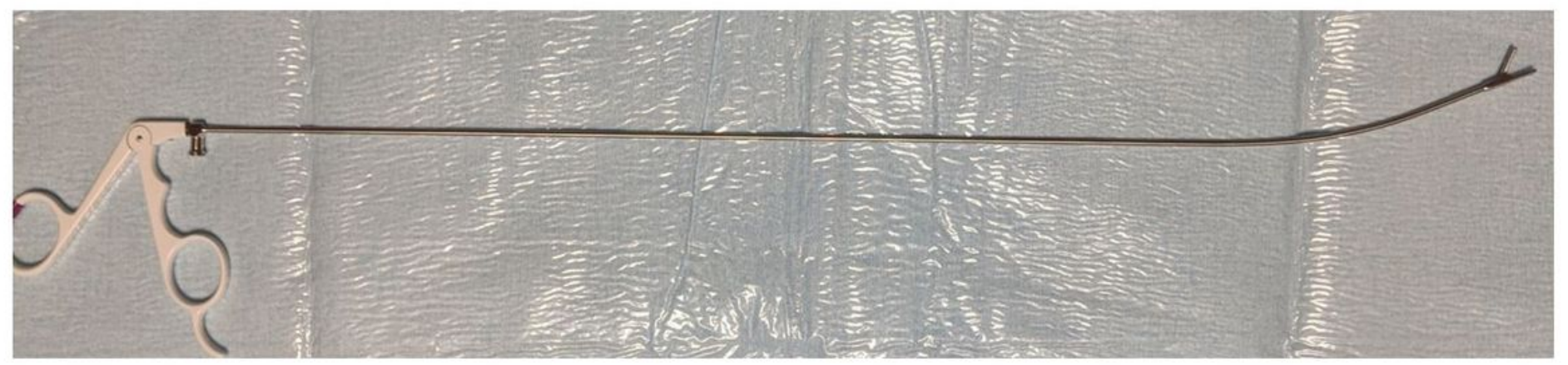

Figure 2

REFs are shapeable and allow for direct grasping of the filter apex. REFs were originally adapted for advance IVCF retrieval, but they can be applied for routine filter retrieval and their ability to be re-used for multiple cases allow for potential procedural cost savings. 